\title{
Scalar Curvature Fluctuations on the Four-Sphere
}

\author{
Sérgio M. C. V. Gonçalves and Ian G. Moss \\ Department of Physics, University of Newcastle Upon Tyne, NE1 7RU U.K.
}

(February 1997)

\begin{abstract}
Two-point functions of the scalar curvature for metric fluctuations on the four-sphere are analysed. The two-point function for points separated by a fixed distance and for metrics of fixed volume is calculated using spacetime foam methods. This result can be used for comparison between the continuum approach to quantum gravity and numerical quantum gravity on the lattice.

Pacs numbers: 03.70.+k, 98.80.Cq
\end{abstract}

Typeset using REVTEX 


\section{INTRODUCTION}

The aim of this paper is to compute two-point functions for the scalar curvature on the 4-sphere. Our results are based upon the de Sitter space graviton propagator found by Allen and Turyn [四]. Curvature two-point functions are also well-suited to evaluation by numerical quantum gravity on the lattice, therefore the result should be useful for comparing two different approaches to quantum gravity [2] 3].

We will use the path integral approach to quantum gravity and constrain the path integral to Euclidean metrics with a fixed volume. This is basically the same setup as the theory of spacetime foam [4. The 4-sphere arises naturally as an instanton in the path integral.

We find that the two-point function $\left\langle R(x) R\left(x^{\prime}\right)\right\rangle$ is a constant plus a $\delta$-function term, which generalizes the known result for flat background [5,6]. This has a simple explanation because, in quantum field theory, we expect expectation values values of field equations to vanish. The field equations for Einstein gravity with a cosmological constant imply that $R=4 \Lambda$, therefore we expect that $\left\langle R(x) R\left(x^{\prime}\right)\right\rangle=16 \Lambda^{2}$ for $x \neq x^{\prime}$.

In a coordinate independent approach to quantum gravity, the usual two-point function looses its meaning and it is preferable to examine the two-point function which has a fixed geodesic distance between the two points. This propagator has a more complicated functional dependence which we calculate below. The analytic result shares the qualitative feature of results from numerical calculations.

\section{THE SCALAR CURVATURE TWO-POINT FUNCTION}

In the Euclidean approach to quantum gravity [1], we examine the quantum fluctuations of the metric $\hat{\mathbf{g}}$ about a classical background $\mathbf{g}$ of signature $(++++)$,

$$
\hat{\mathrm{g}}=\mathbf{g}+2 \kappa \gamma
$$

where $\kappa^{2}=8 \pi G$. The background will be a 4 -sphere of radius $\rho$, related to the cosmological constant by $\rho^{2}=3 / \Lambda$. Vacuum expectation values are defined by Euclidean path integrals.

The graviton propagator $\mathbf{G}$ is defined by

$$
G^{a b c^{\prime} d^{\prime}}\left(x, x^{\prime}\right)=\left\langle 0\left|\gamma^{a b}(x) \gamma^{c^{\prime} d^{\prime}}\left(x^{\prime}\right)\right| 0\right\rangle
$$

It can be decomposed into four components according to its behaviour under traces and divergences [1]

$$
G^{a b a^{\prime} b^{\prime}}=G_{T T}^{a b a^{\prime} b^{\prime}}+G_{T}^{a b a^{\prime} b^{\prime}}+G_{L}^{a b a^{\prime} b^{\prime}}+G_{P T}^{a b a^{\prime} b^{\prime}} .
$$

We will use only the following properties of the various components for contractions with background metric and covariant derivative

$$
\begin{aligned}
G_{P T}^{a b a^{\prime} b^{\prime}} & =-\frac{1}{4} g^{a b} g^{a^{\prime} b^{\prime}} G \\
g_{a b} G_{T T}^{a b a^{\prime} b^{\prime}} & =g_{a b} G_{T}^{a b a^{\prime} b^{\prime}}=g_{a b} G_{L}{ }^{a b a^{\prime} b^{\prime}}=0 \\
\nabla_{a} \nabla_{b} G_{L}^{a b a^{\prime} b^{\prime}} & =\left(\nabla^{a^{\prime}} \nabla^{b^{\prime}}-\frac{1}{4} g^{a^{\prime} b^{\prime}} \nabla^{2}\right) G \\
\nabla_{a} G_{T T}^{a b a^{\prime} b^{\prime}} & =\nabla_{a} \nabla_{b} G_{T}^{a b a^{\prime} b^{\prime}}=0
\end{aligned}
$$


Here, $G\left(x, x^{\prime}\right)$ is a scalar propagator on the 4 -sphere which satisfies

$$
\left(-\nabla^{2}-2 \Lambda\right) G\left(x, x^{\prime}\right)=\delta\left(x, x^{\prime}\right)
$$

It depends only on the geodesic separation $\sigma$ of $x$ and $x^{\prime}$ and is given explicitly by

$$
G\left(x, x^{\prime}\right)=-\frac{1}{2 \pi \rho^{2}} \sec (\pi \nu) F\left(\frac{3}{2}+\nu, \frac{3}{2}-\nu, 2 ; z\right)
$$

where $\nu^{2}=33 / 4$ and

$$
z=\cos ^{2} \frac{\sigma}{2 \rho} .
$$

The first order terms in expansion of the scalar curvature are

$$
R(\hat{g})=R(g)+2 \kappa^{2} \Delta_{a b} \gamma^{a b}
$$

where

$$
\Delta_{a b}=\nabla_{a} \nabla_{b}-\nabla^{2} g_{a b}-\gamma^{a b} R_{a b}(g)
$$

Therefore the connected two-point function is

$$
\left\langle R(x) R\left(x^{\prime}\right)\right\rangle=16 \Lambda^{2}+4 \kappa^{2} \Delta_{a b} \Delta_{a^{\prime} b^{\prime}} G^{a b a^{\prime} b^{\prime}}
$$

From equations (4⿴囗十),

$$
\left\langle R(x) R\left(x^{\prime}\right)\right\rangle=16 \Lambda^{2}+\frac{3}{2} \nabla^{2} \delta\left(x, x^{\prime}\right)+2 \Lambda \delta\left(x, x^{\prime}\right)
$$

This result reduces at $\Lambda=0$ to the result for flat space derived by Modanese [5,6].

\section{THE TWO-POINT FUNCTION OVER A FIXED DISTANCE}

The two-point function over a fixed geodesic proper distance requires a coordinate shift in one of the points to compensate for the metric change in the geodesic length. We do this by moving the second point along the original geodesic.

Consider, for the background metric $g_{a b}$, a geodesic segment $x(t)$, from $x$ to $x^{\prime}$ with proper length given by

$$
\sigma_{g}\left(x, x^{\prime}\right)=\int_{0}^{1} \sqrt{g_{a b} \dot{x}^{a} \dot{x}^{b}} d t .
$$

The tangent vector $\sigma^{a^{\prime}}$ is related to $\sigma_{g}\left(x, x^{\prime}\right)$ by

$$
\sigma^{a^{\prime}}=g^{a^{\prime} b^{\prime}} \frac{\partial \sigma_{g}}{\partial x^{b^{\prime}}}
$$

Now, for the perturbed metric $\hat{g}_{a b}$ the geodesic length changes to 


$$
\sigma_{\hat{g}}\left(x, x^{\prime}\right)=\sigma_{g}\left(x, x^{\prime}\right)+\kappa \int_{0}^{\sigma_{g}} \gamma_{a^{\prime \prime} b^{\prime \prime}} \sigma^{a^{\prime \prime}} \sigma^{b^{\prime \prime}} d s
$$

where $s$ is the proper length along the geodesic to an intermediate point $x^{\prime \prime}$. Hence, one has to introduce an appropriate displacement $\delta x^{a^{\prime}}$, such that

$$
\sigma_{\hat{g}}\left(x^{a}, x^{a^{\prime}}+\delta x^{a^{\prime}}\right)=\sigma_{g}\left(x^{a}, x^{a^{\prime}}\right)=\sigma .
$$

Since we also want to remain in the direction of the original geodesic, we take

$$
\delta x^{a^{\prime}}=\delta x^{\prime} \sigma^{a^{\prime}} .
$$

Combining these equations and using (16) gives

$$
\delta x^{\prime}=-\kappa \int_{0}^{\sigma} \gamma^{a^{\prime \prime} b^{\prime \prime}} \sigma_{a^{\prime \prime}} \sigma_{b^{\prime \prime}} d s
$$

where the integral extends from one end of the geodesic to the other.

The difference between the two-point functions will be denoted by

$$
c\left(x, x^{\prime}\right)=\left\langle 0\left|R(x) R\left(x^{\prime}+\delta x^{\prime}\right)\right| 0\right\rangle-\left\langle 0\left|R(x) R\left(x^{\prime}\right)\right| 0\right\rangle
$$

To first-order in $\delta x^{\prime}$,

$$
c\left(x, x^{\prime}\right)=\left\langle 0\left|R(x) \delta x^{\prime} \sigma^{a^{\prime}} \nabla_{a^{\prime}} R\left(x^{\prime}\right)\right| 0\right\rangle .
$$

This can be computed to order $\kappa^{2}$ using equations (11) and (20),

$$
c\left(x, x^{\prime}\right)=-8 \kappa^{2} \Lambda \int_{0}^{\sigma} d s\left\langle 0\left|\gamma^{a^{\prime \prime} b^{\prime \prime}} \sigma_{a^{\prime \prime}} \sigma_{b^{\prime \prime}} \sigma^{c^{\prime}} \nabla_{c^{\prime}} \Delta_{a^{\prime} b^{\prime}} \gamma^{a^{\prime} b^{\prime}}\right| 0\right\rangle
$$

This simplifies to

$$
c\left(x, x^{\prime}\right)=-\int_{0}^{\sigma} d s \frac{d}{d \sigma} F(\sigma-s)
$$

where

$$
F(\sigma)=8 \kappa^{2} \Lambda \sigma_{a} \sigma_{b} \Delta_{a^{\prime} b^{\prime}} G^{a b a^{\prime} b^{\prime}}\left(x, x^{\prime}\right)
$$

The value of $F(\sigma)$ diverges as $\sigma \rightarrow 0$ and the integral has to be regularised. Performing the integral gives

$$
c\left(x, x^{\prime}\right)=F(\sigma)-F_{0}
$$

where $F_{0}$ is a constant that depends on the regularisation proceedure. We will absorb $F_{0}$ as an order $\kappa^{2}$ correction to $\Lambda$.

For the evaluation of $F(\sigma)$, we use an identity from Allen and Turyn [1],

$$
\left(\nabla^{a^{\prime}} \nabla^{b^{\prime}}-\frac{1}{4} g^{a^{\prime} b^{\prime}} \nabla^{2}\right) G=\rho^{-2}\left(\sigma^{a^{\prime}} \sigma^{b^{\prime}}-\frac{1}{4} g^{a^{\prime} b^{\prime}}\right) z(1-z) \frac{d^{2} G}{d z^{2}}
$$

With equations (47), we obtain

$$
c\left(x, x^{\prime}\right)=-16 \kappa^{2} \Lambda^{2} G(z)-4 \kappa^{2} \Lambda^{2}(1-2 z) \frac{d G}{d z}
$$

This has been plotted in figure 2 . 


\section{SIMPLICIAL QUANTUM GRAVITY}

The analytic result for the two-point function can be compared with numerical results obtained from the method of dynamical triangulations [3], a lattice approach to quantum gravity. In this approach, essentially a modified version of Regge calculus [7], a smooth 4-manifold is approximated by a simplicial manifold built from equilateral 4-simplices with identical edge lengths [8,9]. In addition, the space inside the 4-simplices is flat, with the curvature being concentrated on 2-simplices.

The path integral over metrics on a manifold with spacetime volume $V$ is defined by a partition function

$$
Z\left(N_{4}, k_{2}\right)=\sum e^{k_{2} N_{2}}
$$

where the sum extends over all ways of glueing $N_{4}$ 4-simplices together, such that the resulting complex has the topology of a 4 -sphere and $N_{2}$ is the number of 2-simplices in the triangulation. The parameters relate to the physical volume $V$ and edge length $a$ through

$$
\begin{aligned}
k_{2} & =\sqrt{\frac{3 \pi^{2}}{4}}\left(\frac{a}{\kappa}\right)^{2} \\
N_{4} & =\frac{96}{\sqrt{5}}\left(\frac{V}{a^{4}}\right)
\end{aligned}
$$

The numerical results show different phases, but we concentrate here on the one which has been interpreted as Einstein gravity [3].

In the Euclidean path integral approach to quantum gravity, the partition function at fixed volume is defined by a Laplace transform in terms of the partition function with a cosmological constant, [4],

$$
\begin{aligned}
& Z(V)=\int_{\mathcal{C}} d \Lambda Z(\Lambda) \exp \left(\kappa^{-2} \Lambda V\right) \\
& Z(\Lambda)=\int d \mu[g] \exp \left(-I[g]-\kappa^{-2} \Lambda V[g]\right)
\end{aligned}
$$

where $d \mu[g]$ is the measure, $I[g]$ is the Einstein-Hilbert action and $V[g]$ is the volume of the metric. We will have to make assumptions about the existance of a suitable integral contour $\mathcal{C}$.

To leading order in Planck's constant, $Z(\Lambda)$ is dominated by the 4-sphere. This enables us to express any expectation value $\langle 0|f(\gamma)| 0\rangle_{V}$ obtained from the path integral with constant volume as

$$
\langle 0|f(\gamma)| 0\rangle_{V}=\frac{1}{Z(V)} \int_{\mathcal{C}} d \Lambda\langle 0|f(\gamma)| 0\rangle \exp \left(24 \pi^{2} \kappa^{-2} \Lambda^{-1}+\kappa^{-2} V \Lambda\right)
$$

This integral has a saddle point $\Lambda=\bar{\Lambda}$, where $\bar{\Lambda}^{2}=24 \pi^{2} / V$. The steepest descents contour is a circle centered on the origin. The saddle point gives a good approximation for $V \gg \kappa^{4}$ and in this case any two-point function calculated at constant volume can be replaced by the two-point function on the corresponding 4-sphere. 
Numerical results show a two-point function that begins positive for small distances, becomes negative and then returns positive. The analytic result also has these features. However, from equations (30-31),

$$
\begin{aligned}
\kappa^{2} \bar{\Lambda} & \approx 274.4 k_{2}^{-1} N_{4}^{-1 / 2} \\
\bar{\rho} / a & \approx 0.1725 N_{4}^{1 / 4}
\end{aligned}
$$

The distance scale in figure 2 converted into lattice units differs by a factor $\approx 4$ from the numerical results. It could be that our identification of the bare parameters in the two approaches has been too naive, or that the numerical results are still to far removed from the perturbative regime. It would help resolve this issue if the numerical results were extended to significantly larger numbers of simplices. It would also be interesting to compare the analytic approximation with numerical results for other topologies, particularly $S^{2} \times S^{2}$ which is the next simplest case to the 4-sphere.

\section{ACKNOWLEDGMENTS}

S.G. is supported by the Programa PRAXIS XXI of the J. N. I. C. T. of Portugal. 


\section{REFERENCES}

[1] Allen, B. and Turyn, M. 1987 Nucl. Phys. B 292813

[2] Hamber H. W. 1992 Phys. Rev. D45 507; 1993 Nucl. Phys. B400 347; 1995 Phys. rev. D50 3942.

[3] Smit, J. and Debakker, B. V. 1995 Nucl. Phys. B 454343

[4] Hawking, S. W. 1979 in General Relativity: An Einstein Centenary Survey Eds Hawking, S. W. and Israel, W. Cambridge University Press: Cambridge

[5] Modanese, G. 1992 Phys. Lett. B 28869

[6] Modanese, G. 1994 Riv. Nuovo Cimento 17 No.8 1

[7] Regge, T. 1961 Il Nuovo Cimento XIX No. 3558

[8] Agishtein M. E. and Migdal A. A. 1992 Mod. Phys. Lett. A7 1039

[9] Weingarten D. 1982 Nucl. Phys. B210 229 


\section{FIGURES}

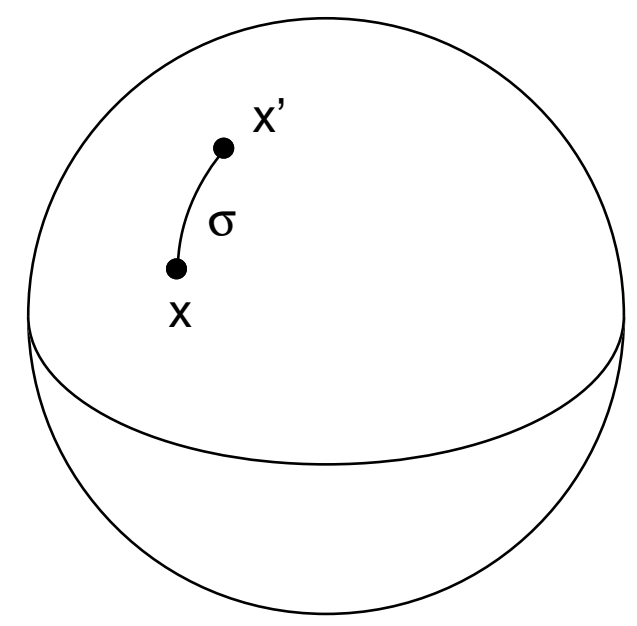

FIG. 1. A geodesic from $x$ to $x^{\prime}$ with the tangent vector $\sigma^{a}$.

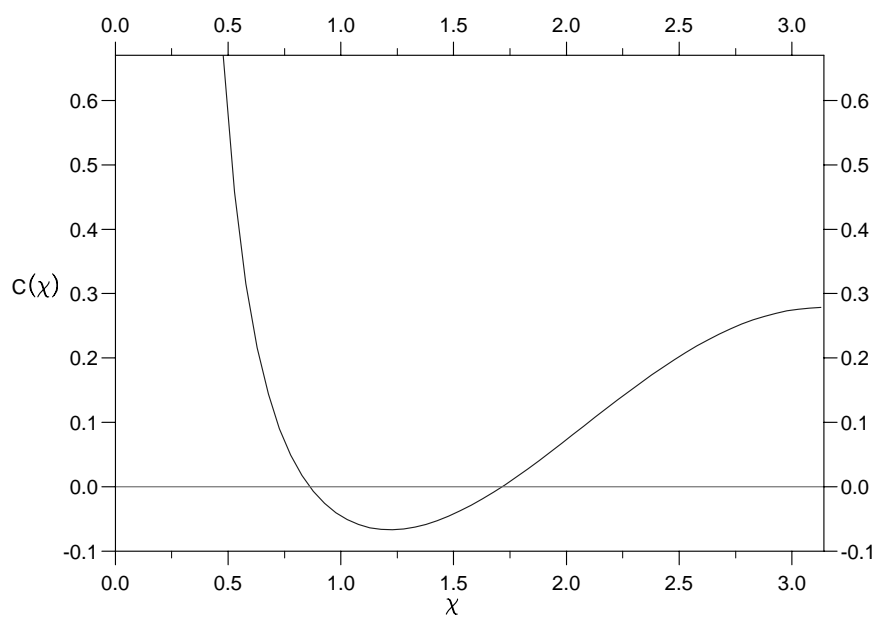

FIG. 2. A plot of the scalar curvature two-point function on the four sphere background with the distance $\sigma$ fixed. $C(\chi)=(\rho / \kappa)^{2}\left(16 \Lambda^{2}\right)^{-2} c\left(x, x^{\prime}\right)$ and $\chi=\sigma / \rho$, where $\rho$ is the radius of the 4-sphere. 